\title{
La experiencia de escucha acusmática: una propuesta de análisis integrado ${ }^{1}$ \\ The Experience of Acusmatic Listening: a Proposal of an Integrative Analysis
}

\author{
por \\ Federico Schumacher Ratti \\ Facultad de Psicología, Universidad Diego Portales, Chile \\ federico.schumacher@gmail.com \\ Claudio Fuentes Bravo \\ Facultad de Psicología, Universidad Diego Portales, Chile \\ claudiofuentesbravo@gmail.com
}

\begin{abstract}
En este artículo proponemos la fundamentación teórica de un conjunto de lineamientos que debiesen ser considerados en la construcción de una metodología de análisis de la experiencia de escucha en música acusmática. Se presenta una consideración crítica de la literatura relevante en el contexto señalado, con el objetivo de señalar principios, directrices o convergencias, que relacionen ciertos enfoques provenientes del análisis musical justificados en su relevancia disciplinar, particularmente aquellos desarrollados por Stéphane Roy y François Delalande, con el marco conceptual que ofrece el campo de la lingüística cognitiva. En la consideración de los casos analizados por Roy y Delalande nos parece evidente que los sujetos al reportar su experiencia de escucha utilizan un conjunto de metáforas cognitivas: en especial, esquemas de imagen y de movimiento ficticios. Los constructos teóricos han sido ya largamente estudiados y descritos a nivel lingüístico como neuroanatómico. Estas metáforas cognitivas son susceptibles de ser utilizadas por auditores de música acusmática durante la descripción de la experiencia de escucha, tanto porque en particular y distintivamente esta música hace un uso intensivo de técnicas de espacialización sonora, como porque todo relato no es otra cosa que el reporte lingüístico de una experiencia de escucha. A partir de lo anterior, postulamos el desarrollo de una metodología multimodal, que nos permita conocer con certeza empírica la experiencia subjetiva de las personas, y al mismo tiempo poder establecer correlatos entre esa experiencia y la traza acústica dada en audición
\end{abstract}

Palabras clave: acusmática, cognición, lingüística, análisis.

We propose a theoretical framework of a set of guidelines for a methodology of analysis of the listener experience in acousmatic music. A critical review of the relevant literature is presented in order to point out principles, guidelines or convergences, in relation to certain approaches from the musical analysis. These are justified in terms of their relevance to the discipline, particularly as relate to the conceptual framework developed by Stéphane Roy and François

${ }^{1} \mathrm{El}$ presente artículo ha sido escrito como parte del proyecto Fondecyt Regular $\mathrm{N}^{\mathrm{o}} 1130620$. Título: "La construcción del contrato musical en la experiencia acusmática: desde las gramáticas de composición hacia la emergencia de una comunidad acusmática”, 2013-2015. 
Delalande, which stems from the field of cognitive linguistics. Considering the cases examined by Roy and Delalande, it seems clear that the subjects reporting their listening experience resort to a set of cognitive metaphors, such as image schemes and fictitious movement. These have been studied and described as theoretical constructs both linguistically and neuroanatomically. The cognitive metaphors are likely to be used by auditors of acousmatic music to describe their listening experience, considering that acousmatic music makes an extensive use of sound spatialization techniques, and that every description is a linguistic report of a listening experience. On the basis of this, we propose the development of a mixed methodology, both qualitative and experimental, designed to ascertain on an empirical basis the subjective experience of the subjects and, at the same time, establish correlations between the experience and the acoustic trace.

Keywords: acousmatic, cognition, linguistics, analysis.

\section{ANÁLISIS DE LA DESCRIPCIÓN DE LA EXPERIENCIA DE ESCUCHA}

"Ninguna escritura ha llegado a dominar el flujo de los detalles de la práctica musical, incluyendo en ella el pensamiento, como representación mental de la música. Siempre permanece un campo sobre el que solo se puede hablar o hacer oír para mostrar y tratar de reproducir esos detalles tan huidizos".

Gustavo Becerra: La posibilidad de una retórica musical hoy (1998: 42).

La capacidad de la música de emocionar, de evocar sentimientos, de representarafectos, de ser el vehículo de un sentido, explícito o implícito, es una de las particularidades acerca de las que se han interesado numerosas disciplinas desde la Antigüedad.

La emergencia en el paisaje cultural occidental durante el siglo XX de las músicas experimentales, acusmáticas e incluso étnicas, han aportado nuevas dimensiones al problema, en la medida de que ellas han contribuido a la desestabilización de un sistema que ligaba, en la producción de sentido, una causa a un efecto, propio de una episteme de tipo funcionalista. La comunicación estaba entonces asegurada en la medida de que el compositor hacía un uso adecuado de unas gramáticas y sintaxis que permitieran la identidad entre la significación propuesta por el compositor y el sentido percibido por el auditor.

Justamente esta identidad es la que ha sido primero puesta en cuestión y luego negada por las teorías actuales no objetivistas. Si entendemos a la música como un objeto simbólico que porta una significación, ello supone un diálogo en el que productor y consumidor, emisor y receptor no son intercambiables, y no tienen por tanto el mismo punto de vista respecto del objeto al que tampoco constituyen de un modo similar, según lo ha establecido Molino (1975). -En esa misma época Nattiez (1975) resaltaba al respecto una cuestión fundamental: los fenómenos musicales son polisémicos, en la medida de que en ellos se produce un reenvío de algo exterior a la obra, que yace en la imaginación de aquellos que la escuchan-.

Entonces, las gramáticas de producción no corresponderían necesariamente a las estrategias de recepción del auditor. La traza acústica solo contiene indicios en donde el auditor desarrolla una lectura personal en función de su propia experiencia. La música, en definitiva, se transforma en un objeto que invita a su interpretación.

Si la significación en música no se encuentra inscrita en la traza acústica, si aquello que calificamos como lo musical no depende exclusivamente del uso de ciertas gramáticas de producción, es entonces en la experiencia de escucha y en la relación entre la traza acústica y algunos auditores donde se encuentra una parte importante, sino esencial, de la producción de sentido en música.

El caso de las músicas acusmáticas, objeto de estudio desde el que presentamos este enfoque analítico, pone particularmente en evidencia los aspectos que acabamos de mencionar, debido a sus características de producción, representación acústica y consumo. En efecto, podemos caracterizar a la música acusmática como un modo de producción musical y como una modalidad de representación acústica de la obra. Si bien algunas de estas características pueden ser compartidas por una amplia variedad de géneros musicales en la actualidad, el conjunto de ellas define las particularidades de este género musical. 
Las características de la acusmática en cuanto modo de producción son las siguientes:

- Se produce en estudio (por ejemplo, en un laboratorio electroacústico).

- Puede utilizar en su producción todos los sonidos posibles, sintéticos o de origen microfónico.

- La representación acústica de la obra acusmática está integrada al proceso de producción.

- Se desarrolla, por tanto, en función de la situación de escucha acusmática.

A su vez, las características de la acusmática en cuanto modalidad de representación acústica son las siguientes:

- Las fuentes sonoras que intervienen en el proceso de representación de la obra surgen únicamente por medio de altoparlantes.

- El dispositivo de altoparlantes está por necesidad distribuido espacialmente, tanto en cuanto a las exigencias de los formatos elegidos durante el proceso de composición como porque la representación se produce en un espacio acústico determinado.

- $\quad$ Es finalmente el dispositivo de altoparlantes el que permite la situación de escucha acusmática ${ }^{2}$.

A causa de la caracterización anterior, dos aspectos, la distribución espacial del dispositivo de altoparlantes, así como la integración de este dispositivo al modo de producción, serán particularmente relevantes para la consideración de la cognición metafórica en el análisis del movimiento espacial, que abordaremos en el punto 4 del presente artículo.

\section{GRAMÁTICAS DE PRODUCCIÓN Y GRAMÁTICAS DE RECONOCIMIENTO}

La literatura teórica pertinente a las maneras posibles de plantear una descripción de la experiencia de escucha es abundante. Verón (1987), por ejemplo, sostiene que el punto de partida de una descripción de operaciones discursivas se encuentra necesariamente siempre del lado de la recepción. En el caso de la acusmática, aquello parece evidente, toda vez que la obra se haya inscrita en un soporte que debe ser transcrito para el desarrollo de cualquier operación analítica. Toda transcripción, no obstante, implica una posición estésica del análisis, ya que hasta el momento no disponemos de transcripciones mecánicas o gráficas al modo de las que planteaba Molino (1975), las que por sí mismas pudieran hacer aparecer el conjunto de datos perceptivos pertinentes que contiene una obra acusmática. Al considerar este problema Delalande (2013) propone desarrollar un método de investigación instalado en el plano estésico, en el que la riqueza de las informaciones recogidas entre los testimonios de escucha (Delalande 2013) pueda ser cruzada con otras variables que forman parte del proceso de significación ${ }^{3}$. En el presente artículo sostenemos que esas variables son fundamentalmente procesos cognitivos corporizados.

2 Para una caracterización más amplia en cuanto a la acusmática en tanto modo de producción y modalidad de representación acústica, ver Schumacher y Fuentes 2016.

3 El mismo Delalande formó parte del equipo que formuló las Unidades Semióticas Temporales (Laboratoire de Musique et Informatique de Marseille, MIM 2002). Este aporte teórico propone 19 unidades descriptivas del significado que puede atribuirse a fragmentos musicales en su evolución en el tiempo. Nuestra aproximación, como se verá en el punto 4, se asienta en las teorías cognitivas de la metáfora, las que han tenido un correlato interesante en relación con la percepción de la espacialidad en música acusmática, principalmente en Smalley (2007) y Kendall (2010). 
Compartiendo objetivos similares, aunque difiriendo en los supuestos teóricos, la Teoría Generativa de la Música Tonal (Lerdahl y Jackendoff 1983) se basa en enfoques cognitivos para desarrollar una gramática de producción basada en la percepción. Vale decir, una gramática de composición que depende de las gramáticas auditivas (listening grammars), las que se definen como aquellas que "son empleadas más o menos conscientemente por los auditores y que engendran representaciones mentales de la música” (Lerdahl 1989: 28).

La existencia de estas gramáticas supone al menos tres condiciones:

- Un auditor experimentado.

- La organización psicoacústica de la señal física según una superficie musical (traza acústica) constituida por una secuencia de eventos sonoros.

- Como una asimilación final resultante de la secuencia, la teoría anticipa descripciones estructurales de superficies musicales, mediante un conjunto de normas que corresponden, idealmente, a la gramática auditiva.

Es importante considerar que esta teoría fue desarrollada para y dentro del espacio restrictivo de la música tonal. No obstante lo anterior, se advierten entre las condiciones mencionadas ciertos aspectos que dificultan la posibilidad de una identidad entre gramáticas de reconocimiento y de composición. Tal es el caso de la música acusmática y, probablemente, de todas aquellas músicas que desbordan el espacio de la tonalidad o de la notación prescriptiva:

- La idea de auditor experimentado o auditor ideal no toma en cuenta las diversas estrategias y conductas de escucha (Delalande 1987, 1989, 2013; Alcázar 2004). Por tanto, si lo que se espera de la audición de un auditor son descripciones estructurales de la traza acústica, se necesitaría una estrategia de escucha taxonómica. Usualmente se asume que este tipo de estrategia provenga de profesionales de la música, particularmente compositores. No obstante, tampoco estos últimos desarrollan estrategias de escucha exclusivamente taxonómicas (Delalande 2013). En las experiencias que permitieron a Delalande establecer las diferentes estrategias de escucha (taxonómica, empática y figurativa), la muestra estaba configurada básicamente por compositores pertenecientes al Grupo de Investigaciones Musicales (Groupe de Recherches Musicales, GRM).

- La pertinencia cognitiva de las gramáticas de composición se da en relación con la especificidad de la traza acústica, su modalidad de producción, sus condiciones de escucha y un conjunto de aspectos que conforman las gramáticas de producción y reconocimiento. Ahora bien, lo que es pertinente en una música tonal para piano representada en una situación de concierto, no lo es necesariamente en una música para una "rave", por ejemplo, y por supuesto tampoco lo es para la música acusmática. En este último caso se han introducido variables perceptivas y cognitivas que surgen del desarrollo tecnológico y del dispositivo de escucha, particularmente el uso sistemático del movimiento espacial de los sonidos, tanto durante la producción de la obra como en su representación acústica. Aunque el impacto respecto de la percepción y en las gramáticas de composición acusmáticas de estos procedimientos no ha sido aún estabilizado y sistematizado, es innegable que ellos juegan un rol importante en la producción de sentido.

- No obstante lo anterior, no se puede afirmar que exista hoy un corpus teórico formalizado que pudiéramos llamar una gramática de composición acusmática como tal. Lo que existe son prácticas más o menos sistemáticas de las que no conocemos con exactitud 
su impacto sobre la percepción o su pertinencia cognitiva. Sin embargo estas prácticas parecen significativas en el proceso de producción de sentido. De un cierto modo, en el caso de la música acusmática podría darse el caso de la existencia de gramáticas de reconocimiento, que buscan establecer relaciones con una gramática de producción.

En consecuencia, es en la experiencia de escucha del auditor donde nos parece pertinente buscar una interpretación cognitiva de los fenómenos sonoros implicados en el proceso de significación de la música acusmática.

\section{PRIMEROS PASOS HACIA UN ENCUADRE COGNITIVO DEL ANÁLISIS DE LA SITUACIÓN DE ESCUCHA ACUSMÁTICA}

En su libro acerca del análisis de las músicas electroacústicas, Stephan Roy (2003) desarrolla una metodología que denomina como Análisis funcional. Este método conformado por datos cualitativos propone una matriz formada por cuarenta y cinco funciones divididas en cuatro categorías: de orientación, estratificación, proceso y retórica. Estas "permiten conceptualizar el rol que juegan dentro de la obra ciertas unidades musicales pertinentes" (Roy 2003: 341).

Roy pretende extraer las posibles convergencias mediante un número idealmente restringido de figuras retóricas. En la medida en que estas figuras "son procedimientos expresivos que operan por relaciones entre unidades (reenvío, oposición) o simplemente por efectos de ruptura en el flujo musical" (Roy 2003: 348), entonces pueden servir de referente para la interpretación y comprensión del significado y sentido atribuido por el auditor a una traza acústica.

En este enfoque las figuras son metáforas sobre "metáforas comparables", según señala François Delalande en su análisis de Sommeil (2013: 44).

Entre estas categorías, la de retórica contiene quince figuras divididas en:

- Retórica relacional: llamada, anuncio, tema, anticipación, afirmación, reiteración, imitación, antagonismo simultáneo o sucesivo.

- Retórica de la ruptura: desviación, paréntesis, indicio, articulación, retención, ruptura, espacialización.

Por el momento estas figuras conforman el repertorio inicial de unidades interpretativas disponibles, pudiendo incorporarse algunas o suprimirse otras mediante la realización de nuevos análisis descriptivos.

Las experiencias que permitieron a François Delalande y Jean François Thomas plantear la existencia de diversas estrategias de escucha o conductas-tipo, se realizaron con las siguientes obras:

- Sommeil, primer movimiento de las Variations pour une porte et un soupir de Pierre Henry (de 1979 a 1988 con Jean Christophe Thomas).

- La Terrasse des audiences du clair de lune de Claude Debussy por Cécile Ousset (1989, con Jean Christophe Thomas).

- Aquatisme, extracto de La création du monde de Bernard Parmegiani (1991, con Jean Christophe Thomas y Dominique Besson).

- La musique électroacoustique (CD rom) sección entendre: six extraits ou mouvements de musiques électroacoustiques (obras de Ake Parmerud, Bernard Parmegiani, Philippe Leroux, Pierre Henry, Alain Savouret y François Bayle) (1999-2000).

- $\quad$ Le Plein du vide de Xu Yi (2006, con Maÿlis Dupont). 
A partir de estas experiencias, los autores desarrollaron un procedimiento metodológico al que denominaron "testimonios de escucha". Consiste, brevemente, en que los sujetos son invitados a escuchar individual y confortablemente una obra o fragmentos de ella. Luego de cada audición los sujetos testimonian con total libertad su experiencia de escucha a partir de dos preguntas: ¿Qué ha escuchado? y ¿Cómo lo ha escuchado? Los testimonios son grabados, transcritos y finalmente comparados. De este último ejercicio se obtienen distinciones metafóricas que pueden ser comparables.

No obstante, el mismo Delalande (2010) plantea algunas prevenciones sobre los límites de la verbalización acerca de la vivencia de la experiencia:

- El hecho de tener que relatar la experiencia influye en la estrategia de escucha (como cualquier otro condicionamiento).

- El relato de una audición es una reconstrucción, lo que implica ausencias y desarrollos. Por esta razón, el relato no puede ser considerado una imagen fiel de lo que ocurrió durante la experiencia de escucha.

- El análisis comparativo de los testimonios transcritos conduce a reagrupar verbalizaciones análogas pero no idénticas, lo que conlleva aproximaciones e interpretaciones.

\subsection{Un ejercicio interpretativo}

¿Es posible realizar una lectura de los testimonios de escucha en función de las figuras retóricas propuestas?

A continuación presentaremos un ejercicio interpretativo de algunas de las citaciones de testimonios de escucha que consigna Delalande en el capítulo "Análisis musical y conductas de recepción: Sommeil de Pierre Henry" de su libro Analyser la musique, pourquoi, comment? (2013). Considerando la advertencia de Roy (2003: 349), con relación a que el análisis retórico más que cualquiera de las otras categorías funcionales necesita de las facultades de interpretación del analista, el ejercicio que presentamos tiene limitaciones evidentes, pues no disponemos de los registros sonoros originales necesarios para exponer formalmente la vinculación entre el texto citado y el segmento de la traza acústica con la que se relaciona. No obstante esta limitación, el objetivo fundamental del ejercicio es mostrar la viabilidad teórica de la propuesta analítica integrativa que planteamos.

\section{TABLA 1}

\begin{tabular}{|c|}
\hline Ejemplo 1 \\
\hline Figura retórica: Espacialización. \\
\hline Definición: "Esta función aparece en unidades donde la variación en el espacio interno de \\
la obra presenta rupturas repentinas de localización espacial. Esta localización puede ser \\
real, gracias a desplazamientos cinéticos de las unidades en un espacio tridimensional de \\
concierto (arriba/abajo, adelante/atrás, izquierda/derecha, según el posicionamiento de \\
los altoparlantes), desplazamientos preliminarmente fijados en la obra (y no creados en \\
tiempo real por el intérprete desde una consola). La localización puede igualmente ser \\
estimulada por un tratamiento sobre el timbre gracias a efectos de reverberación" (Roy \\
2003: 363). \\
\hline
\end{tabular}




\begin{tabular}{|c|c|}
\hline \multicolumn{2}{|l|}{ Testimonios } \\
\hline \multirow[t]{2}{*}{$\begin{array}{l}\text { Estrategia taxonómica: Este tipo de escucha se manifiesta } \\
\text { por una tendencia del auditor a desarrollar distinciones, } \\
\text { segmentaciones y a cualificar la traza acústica, con el objeto } \\
\text { de obtener una mirada sinóptica de la obra } \\
\text { (Delalande 2013: 46). }\end{array}$} & $\begin{array}{l}\text { "Auditor C: Los planos, las } \\
\text { presencias: el golpe agudo } \\
\text { está manifiestamente en un } \\
\text { plan de presencia más lejano } \\
\text { (reverberación, espacio artificial, } \\
\text { alejamiento), incluso si su ataque } \\
\text { está cercano. La respiración está } \\
\text { completamente próxima. El } \\
\text { otro golpe, grave, regular (que } \\
\text { no se escucha hasta el final) } \\
\text { está prácticamente a nivel de la } \\
\text { membrana (del altoparlante)" } \\
\text { (Delalande 2013: 49). }\end{array}$ \\
\hline & $\begin{array}{l}\text { "Auditor F: En ese momento el } \\
\text { soplido tiene otro color (...) Y } \\
\text { tengo la impresión que se acerca } \\
\text { (...) La gota de agua la percibo } \\
\text { siempre del mismo modo: al } \\
\text { fondo (esta gota de agua tiene } \\
\text { una resonancia)" } \\
\text { (Delalande 2013: 49). }\end{array}$ \\
\hline \multirow{2}{*}{$\begin{array}{l}\text { Estrategia empática: El auditor que adopta esta actitud } \\
\text { está atento a las sensaciones, que declara sin tapujos como } \\
\text { "fisiológicas", que produce el sonido en él. Los comentarios } \\
\text { se relacionan entonces al nivel de la sensación corporal } \\
\text { (Delalande 2013: 69). }\end{array}$} & $\begin{array}{l}\text { "Auditor E: Algo que cae desde } \\
\text { arriba, vertical, se impone. Golpe } \\
\text { de punzón, de martillo, que } \\
\text { hunde hacia algo que frota pero } \\
\text { que avanza de todas maneras; } \\
\text { algo que barre" } \\
\text { (Delalande 2013: 59). }\end{array}$ \\
\hline & $\begin{array}{l}\text { "Auditor E: El barrido, lo percibo } \\
\text { como yendo en un sentido } \\
\text { horizontal y el resto, tengo } \\
\text { la impresión que es algo que } \\
\text { cae desde arriba... en sentido } \\
\text { vertical" (Delalande 2013: 62). }\end{array}$ \\
\hline $\begin{array}{l}\text { Estrategia figurativa: Este tipo de escucha corresponde a una } \\
\text { tendencia en el auditor a encontrar algo "figurativo" en la } \\
\text { música, más precisamente a considerar que al menos ciertos } \\
\text { sonidos evocan algo que tiene movimiento, eventualmente, } \\
\text { que vive (Delalande 2013: 70). }\end{array}$ & $\begin{array}{l}\text { “Auditor C: (...) Es la gota } \\
\text { de agua, la llave que gotea } \\
\text { mientras uno duerme, es algo } \\
\text { que marca el tiempo (...) Es el } \\
\text { elemento exterior, un sonido } \\
\text { exterior (...) Los otros dos, } \\
\text { eso sería el interior". "Dos } \\
\text { elementos sin altura, interiores } \\
\text { (La 'respiración' y el 'corazón' } \\
\text { contra [sonido] tónico, exterior. } \\
\text { (la señal 'gota de agua')" } \\
\text { (Delalande 2013: 70). }\end{array}$ \\
\hline
\end{tabular}


Lo que comenzamos a definir, en términos generales, es la posibilidad de describir en términos de un enfoque cognitivo del lenguaje, lo que Roy ha definido como una figura de espacialización. En la tabla 1, la exposición se ordena mostrando el ejemplo de una figura de espacialización. Se la define en primer lugar a partir de sus cualidades acústicas y perceptivas. En las filas que continúan podemos encontrar los testimonios de los auditores "C", "E" y "F". Más adelante en la columna de la izquierda se caracterizan las estrategias de escucha "taxonómica", "empática” y "figurativa”. La "estrategia taxonómica" define el testimonio de los auditores "C" y "F" cuyo contenido se resume en la columna a la derecha de la tabla 2; luego, la "estrategia empática" define el testimonio del auditor " $\mathrm{C}$ " en dos instancias, los que se pueden leer en la columna respectiva de la tabla 3; y, finalmente, la "estrategia figurativa" que definiría el testimonio de "C" cuyo contenido aparece, como en los casos anteriores, en la columna a la derecha de la tabla 4, en la que se agregan las características espectromorfológicas que definen acústicamente al sonido evocado.

TABLA 2

ESTRATEGIA DE ESCUCHA TAXONÓMICA

\begin{tabular}{|c|c|}
\hline Sonido & Cualificación \\
\hline Golpe agudo & Lejano \\
\hline Respiración & Muy próxima \\
\hline Golpe grave regular & A la altura del altoparlante \\
\hline
\end{tabular}

TABLA 3

ESTRATEGIA DE ESCUCHA EMPÁTICA

\begin{tabular}{|c|c|}
\hline Acción & Expresión asociada \\
\hline Descripción del movimiento & Algo cae desde arriba de modo vertical \\
\hline Metaforización (1) (¿cómo sueña?) & Suena como un golpe de punzón o de martillo \\
\hline Metaforización (1.1) (qué hace eso que suena) & Se hunde, frita, avanza, barre \\
\hline
\end{tabular}

TABLA 4

ESTRATEGIA DE ESCUCHA FIGURATIVA

\begin{tabular}{|c|c|c|}
\hline $\begin{array}{c}\text { Descripción del } \\
\text { espacio }\end{array}$ & Expresión asociada & $\begin{array}{c}\text { Características } \\
\text { espectromorfológicas del sonido } \\
\text { evocado }\end{array}$ \\
\hline Espacio exterior & $\begin{array}{c}\text { "Es la gota de agua, la llave que } \\
\text { gotea mientras uno duerme, } \\
\text { es algo que marca el tiempo" } \\
\text { (Delalande 2013: 49) }\end{array}$ & $\begin{array}{c}\text { Sonido tónico: Sonido cuya masa es } \\
\text { percibida como condensada en un } \\
\text { punto de la tesitura, es decir, que } \\
\text { tiene una altura que responde a la } \\
\text { definición tradicional de la nota de } \\
\text { música (Schaeffer 1966: 466) }\end{array}$ \\
\hline
\end{tabular}




\begin{tabular}{|c|c|c|}
\hline $\begin{array}{c}\text { Descripción del } \\
\text { espacio }\end{array}$ & Expresión asociada & $\begin{array}{c}\text { Características } \\
\text { espectromorfológicas del sonido } \\
\text { evocado }\end{array}$ \\
\hline Espacio interior & $\begin{array}{c}\text { "Los dos otros, eso sería el interior". } \\
\text { (la respiración y el corazón )" } \\
\text { (Delalande 2013: 70) }\end{array}$ & $\begin{array}{c}\text { Sonido complejo: La masa parece } \\
\text { fija en tesitura, sin poder ser } \\
\text { claramente establecida una altura } \\
\text { (Schaeffer 1966: 466) Sonidos a los } \\
\text { cuales no puede atribuírsele una } \\
\text { altura nominal precisa pero que } \\
\text { son susceptibles de ser analizados } \\
\text { como más o menos agudos, medios } \\
\text { o graves (Chion 1983: 145). }\end{array}$ \\
\hline
\end{tabular}

\section{DISCUSIÓN: ANÁLISIS FUNCIONAL Y METÁFORA COGNITIVA4}

La concepción del lenguaje inscrita en la perspectiva experiencialista asume dos supuestos centrales (Lakoff, 1987; 1988; 1990): (1) la existencia de una sistema conceptual, constituido a partir de la experiencia corporal y social del ser humano; (2) el ejercicio de la facultad imaginativa, esto es, la capacidad para proyectar realidades mentales a partir de hechos físicos o datos empíricos. Ambos supuestos están palmariamente presentes en los procesos de metaforización descritos por los auditores $\mathrm{C}$, E y F, que se recogen en las tablas anteriores.

La filosofía ha trabajado tradicionalmente con casos prototípicos como "El gato está en la alfombra o Sally le da el libro a John" (Núñez, 2010). Si usamos en cambio expresiones cotidianas, nos encontraremos con afirmaciones como "Envíale mis más cálidos saludos" (Núñez 2010). La pregunta que se plantea Núñez es de central importancia: ¿Cómo hablamos de afectos, tiempo, de cosas que no percibimos directamente? Es decir, si pensamos en lo que dice el auditor C (Tabla 1), podemos adaptar la pregunta de Núñez a: ¿Cómo el auditor $\mathrm{C}$ habla de su experiencia de espacialización? La lingüística cognitiva ha estudiado estos fenómenos principalmente mediante la noción de metáfora.

A continuación pasaremos revista a dos mecanismos cognitivos especialmente pertinentes a los procesos de metaforización, que nos parece se ponen en acción en los ejemplos de las tablas 2 y 3 .

\subsection{Movimiento ficticio}

Talmy $(1996,2000)$ observó que comúnmente hablamos (inconscientemente y sin esfuerzo, pero con precisión) acerca de objetos estáticos (literales o metafóricos) como si fueran dinámicos. Este fenómeno se manifiesta a sí mismo en el lenguaje, y tiene una estructura de lo que es llamado ir de un lado a otro. Un ejemplo que propone Núñez (2010) es "La reja termina luego del árbol”. La reja no se mueve, es un objeto estático, pero lo describimos como si se moviera.

La evidencia que respaldaría estas afirmaciones es la teoría de mapeos conceptuales que se ha desarrollado en estos últimos años, reuniendo evidencia empírica en diferentes

4 Describimos más ampliamente nuestro enfoque a propósito de la cognición musical y la acusmática en el texto "Doble articulación y ciencias cognitivas de tercera generación: desafiando las nociones tradicionales en torno a la experiencia acusmática" (Schumacher y Fuentes 2016). 
dominios por medio de estudios con electroencefalografía, $\mathrm{fMRI}^{5}$, priming ${ }^{6}$, y producción gestual. Núñez (2010) describe en las "Shanghai Lectures" los experimentos realizados en su laboratorio al respecto, en los que se han comparado dos expresiones metafóricas que aparentemente significan lo mismo, como "Te estás acercando a la Navidad" y "La Navidad se está acercando".

El estudio realizado consistió en utilizar técnicas de fMRI en una habitación totalmente oscura, en donde se enviaron señales auditivas a sujetos experimentales, y se les hizo escuchar las oraciones referidas en el párrafo anterior. Lo que resultó fue un patrón diferente e interesante entre estas dos expresiones metafóricas que aparentemente significan lo mismo, pero que en un nivel neuroanatómico son muy distintas.

El área visooccipital del cerebro se mostró especialmente activa cuando los sujetos escucharon "ver la Navidad acercarse". Por otra parte, la zona polisensorial también sería especialmente receptiva a "cosas que se mueven", no solo cuando efectivamente algo se acerca a nuestra cara o brazos, sino cuando el movimiento es figurativo, como en el caso del experimento de Núñez con oraciones del tipo "la Navidad se acerca". Queda por determinar en futuras investigaciones si lo que es percibido como un "sonido que se mueve" por los auditores C, E y F pueda corresponder a un fenómeno de movimiento ficticio en los términos en los que ha sido descrito por Talmy (1996, 2000), Núñez y Lakoff (2000); así como en Nuñez (2010).

\subsection{Esquemas de imagen}

Un esquema es una representación cognitiva de hechos, percepciones, relaciones u objetos, cuyas propiedades son generalizadas a partir de múltiples instancias u ocurrencias (Osorio 2011: 220). Es un patrón recurrente, una figura, "que, por tanto, contribuye a la regularidad, coherencia y comprensibilidad de nuestra experiencia y entendimiento" (Johnson y Rohrer 1987: 127).

Estos patrones emergen como estructuras significativas para nosotros, principalmente en el nivel de nuestros movimientos corporales en el espacio, nuestra manipulación de objetos y nuestras interacciones perceptuales. Son entendidos como conceptos de alto nivel y su configuración es independiente del contexto. En tanto conceptos, operan en diferentes dominios y son capaces de darle forma y significado a la experiencia. Los esquemas son entendidos como estructuras estables y persistentes en el sistema cognitivo (Osorio 2011: 221).

Entre los esquemas de imagen que Johnson propone se cuentan, entre otros, los siguientes: contenedor, coacción, capacitación, atracción, vínculo, centro-periferia, partetodo, ciclo, superficie, proceso (Osorio 2011: 221).

El esquema contenedor (Lakoff 1989), por ejemplo, consiste en una distinción de límites entre un interior y un exterior. Define la distinción más básica entre dentro y fuera, así como hace el auditor C en el ejemplo de estrategia de escucha figurativa (tabla 4), a la que asociamos las características acústicas y espectromorfológicas del sonido evocado según las definiciones de la tipomorfología schaefferiana. Esta distinción entre interior y exterior parece esencial en cuanto a la percepción espacial de fenómenos sonoros y es a partir de ellas que Kendall (2010) propone la noción de esquemas espaciales -esquemas recurrentes por los que comprendemos el comportamiento del sonido en el espacio-. Durante la audición de estímulos sonoros provenientes de un dispositivo de escucha de tipo

5 Sigla en inglés para Resonancia Magnética Funcional.

6 Priming es un efecto relacionado con la memoria implícita en el cual la exposición a un estímulo influencia la respuesta a otro estímulo, presentado con posterioridad. Este fenómeno puede ocurrir a nivel perceptivo, semántico o conceptual. 
electroacústico-acusmático, donde no hay otras informaciones sensoriales que lo auditivo, estos esquemas dan coherencia a la información espacial percibida, la que de otro modo sería débil o incompleta. Los esquemas espaciales parecen ser un correlato auditivo de los esquemas visuales, lo que podemos apreciar en las tablas 2, 3 y 4.

Ahora bien, en el paradigma cognitivo corporizado todo esquema de imagen remitiría, en último término, a una experiencia física, la que en este caso se relaciona con el hecho de asumir nuestros propios cuerpos como contenedores, mediante la experiencia más básica todavía de ingerir y excretar, tomar aire y respirar.

Johnson y Rohrer (2007), junto con apostar a un correlato neurológico de estas estructuras, postulan que el carácter habitualmente no consciente de los esquemas de imagen puede dar paso a situaciones en las que el esquema es activado conscientemente como una estructura de la experiencia.

De este modo la naturaleza preverbal y no consciente de los esquemas de imagen, jugaría un papel relevante en la identificación de relaciones conceptuales significativas y en la producción de expresiones nuevas motivadas por esas mismas relaciones. Si asumimos que algunas de nuestras experiencias físicas pueden llegar a ser conscientes, en términos de esquemas de imagen, estas estructuras se perfilarían como patrones que inciden directamente en la asignación de un significado lingüístico.

Para el caso del ejemplo de la tabla 3, que recordamos aquí, es bastante clara por todo lo dicho la pertinencia de los esquemas para describir las acciones del agente que se señala en la tabla 5 .

TABLA 5

\begin{tabular}{|c|c|}
\hline Acción & Expresión asociada \\
\hline Descripción del movimiento & Algo cae desde arriba de modo vertical \\
\hline Metaforización (1) (¿cómo suena?) & Suena como un golpe de punzón o de martillo \\
\hline Metaforización (1.1) (¿qué hace eso que suena?) & Se hunde, frota, avanza, barre \\
\hline
\end{tabular}

\section{CONCLUSIONES}

La tesis tradicional en la música asume que el uso de unas gramáticas y sintaxis adecuadas permiten la identidad entre la significación intentada por el compositor y el sentido percibido por el auditor. Las teorías contemporáneas no objetivistas han negado esta identidad.

En la línea de lo anterior, Delalande (2013) propone desarrollar un método de investigación basado en los testimonios de escucha vinculados a variables que forman parte del proceso de significación. En el presente artículo hemos sostenido que esas variables son fundamentalmente procesos cognitivos corporizados.

Roy (2003) pretende extraer las posibles convergencias en los juicios de los sujetos (analistas) mediante su clasificación en un número idealmente restringido de figuras retóricas. De este ejercicio se obtienen distinciones metafóricas que pueden ser comparables.

En tanto el testimonio sea el producto lingüístico de una experiencia de escucha, la estrategia expresiva que utiliza el sujeto para describir sus estados mentales suele ser metafórica. Pues bien, el análisis de las metáforas es un análisis indirecto de la experiencia del auditor de música acusmática. Tenemos la pieza acusmática, luego respecto de ella se realiza la experiencia de escucha de esa pieza, luego el testimonio lingüístico de esa experiencia de escucha, y finalmente el análisis del testimonio. No se trata, como se puede constatar, de un análisis de la experiencia en cuanto tal, sino del testimonio lingüístico de la experiencia. 
El análisis de la experiencia de escucha es de algún modo un análisis del discurso en tanto este expresa metafóricamente una experiencia particular. Dicho lo anterior, parece pertinente vincular la metaforización de la experiencia en la propuesta analítica de Roy con tres constructos teóricos de la lingüística cognitiva: el movimiento ficticio, los esquemas de imagen y los esquemas espaciales. Los enfoques analíticos mencionados parecen de especial relevancia para el caso de la música acusmática, en función de la importancia que adquiere la espacialidad, tanto en cuanto a sus condiciones de producción como de representación acústica.

Hemos postulado que los esquemas analíticos desarrollados por Roy son susceptibles de ser interpretados como esquemas cognitivos particulares, para ello nuestra propuesta es desarrollar una metodología multimodal (Hernández Sampieri, Fernández y Baptista 2004), que nos permita conocer con certeza empírica la experiencia subjetiva del agente. De especial relevancia en este contexto nos parece la metodología fenomenológica propuesta por Delalande, la que puede ser complementada con estudios neurofisiológicos.

El desafío investigativo futuro, entonces, parte de la constatación de los resultados experimentales de Núñez (2010), quien nota especialmente activas las áreas visooccipital y polisensorial del cerebro con "cosas que se mueven", no solo cuando efectivamente algo se acerca a nuestra cara o brazos, sino cuando el movimiento es figurativo, con oraciones del tipo "la Navidad se acerca".

Parece razonable pensar que un "sonido que se mueve", como en el caso de la experiencia de los auditores $\mathrm{E}$ y $\mathrm{F}$ de la tabla 1 pueda corresponder a un fenómeno de movimiento ficticio en los términos que han sido descritos por Talmy (1996, 2000), Núñez y Lakoff (2000) y por Núñez (2010), y por tanto encuentre una explicación análoga en términos neurofisiológicos.

Finalmente, al disponer del conjunto de informaciones obtenidas, tanto descriptiva como experimentalmente para el análisis orientado específicamente a la música acusmática, se puede asumir, a partir de la experiencia de la escucha acusmática, que ha sido objeto de diferentes procesos cognitivos: memoria, percepción, emoción. Sobre esta base se podría volver a la traza acústica para intentar descubrir qué aspectos de lo que suena se relacionarían con lo que ha sido descrito, de modo de comenzar a prefigurar una posible gramática cognitiva de composición acusmática.

\section{BIBLIOGRAFÍA}

\section{Alcázar Aranda, Antonio}

2004 Análisis de la música electroacústica-género acusmático-a partir de su escucha. Tesis para obtener el grado de doctor en Musicología. Cuenca: Universidad de Castilla- La Mancha, Facultad de Bellas Artes. Directores: José Antonio Sarmiento y Francisco González.

BECERRA-Schmidt, Gustavo

1998 "La posibilidad de una retórica musical hoy", Revista Musical Chilena, LII/189 (enero-junio), pp. 37-52.

Chion, Michel

1983 Guide des objets sonores. Paris: INA-GRM/Buchet-Chastel.

Delalande, François

1987 "L'analyse des conduites musicales: Une étape du programme sémiologique?", Semiotica, LXVI-1/ 3 (enero), pp. 99-107. 
1989 "La terrasse des audiences du clair de lune: un essai d'analyse esthésique”, Analyse Musicale. 16 (octubre), pp. 3-23.

2010 Signification et émotion en musique. No publicado. Archivo personal del autor.

2013 Analyser la musique, pourquoi, comment? Paris: INA.

Graziano, Michael; Xin Tian Hu, Gross, Charles

1997 "Coding the Locations of Objects in the Dark", Science, XI/277 (julio), pp. 239-241.

Hernández Sampieri, Roberto; Fernández, Carlos y Baptista, Pilar

2004 Metodología de la Investigación. México: McGraw-Hill.

Johnson, Mark y Rohrer, Tim

2007 "We are Live Creatures: Embodiment, American Pragmatism, and the Cognitive Organism”, en Tom Ziemke, Zlatev Jordan, Ross Frank y René Dirven (editores). Body, Language, and Mind. Volumen I. Embodiment. Berlin: Mouton De Gruyter, pp. 17-54.

KENDALL, GARY

2010 "Spatial Perception and Cognition in Multichannel Audio for Electroacoustic Music", Organised Sound, XV/3 (diciembre), pp. 222-238.

Laboratoire Musique et Informatioue de Marseille (MIM)

2002 Les Unités Sémiotiques Temporelles: Nouvelles clés pour l'écoute. CD-ROM. Marseille: MIM.

LAKOFF, GEORGES

1987 Women, Fire, and Dangerous Things. Chicago: University of Chicago Press.

1988 “Cognitive Semantics”, en Umberto Eco (editor). Meaning and Mental Representation. Bloomington: Indiana University Press. pp. 119-153.

1990 "Some Empirical Results About the Nature of Concepts", Mind and Language, IV/1-2 (primavera-verano) pp. 103-109.

LAKofF, Georges y Johnson, Mark

1980 Metaphors we Live by. Chicago: University of Chicago Press.

LERDAHL, FRED y JACKeNDOFF, RAY

1983 A Generative Theory of Tonal Music. Cambridge: MIT Press.

LERDAHL, FRED

1989 "Contraintes cognitives sur les systèmes compositionnels", Contrechamps, $\mathrm{N}^{\circ} 10$, Composition et perception. Lausanne: Editions l'Age d'Homme. pp. 25-57.

MoLino, JEAN

1975 Le singe musicien. Paris: Actes-Sud.

Nattiez, Jean Jacques

1975 Fondements d'une sémiologie de la musique. Paris: Union Générale d'Édition.

NuÑEZ, RAFAEL

2010 Building up Evidence for the Lakoff-Nuñez Hypothesis. Recuperado desde http:/ / shanghailectures.org/guest-lectures/nunez-where-mathematics-comes-from, el $3 / 07 / 2017$.

NúÑEZ, Rafael y LakofF, GEORGE

2000 Where Mathematics comes from: How the Embodied Mind brings Mathematics into Being. Nueva York: Basic Books. 
OSORIO, JORGE

2011 "Metáfora y esquema de imagen en un caso de construcción idiomática", en Cristián Santibáñez y Jorge Osorio (editores). Recorridos de la metáfora: mente, espacio y diálogo. Concepción: Cosmigonon. pp. 217-239.

Roy, STEPHAN

2003 L'analyse des musiques électroacoustiques: modèles et propositions. Paris: L'Harmattan.

SchaefFer, Pierre

1966 Traité des objets musicaux. Paris: Seuil.

Schumacher Ratti, Federico y Claudio Fuentes Bravo

2016 "Doble articulación y ciencias cognitivas de tercera generación: desafiando las nociones tradicionales en torno a la experiencia acusmática”, Resonancias, XX/39 (julio-noviembre) pp. 93-111.

Smalley, Denis

2007 "Space-Form and the Acousmatic Image", Organised Sound, XII/1 (abril), pp. 35-58.

TALMY, LEONARD

1996 Fictive Motion in Language and 'Ception'. Language and Space. Cambridge: The MIT Press.

2000 Towards a Cognitive Semantics. Cambridge: The MIT Press.

VERón, Eliseo

1987 La semiosis social. Barcelona: Gedisa. 